\title{
Gauge Theory's Expansion in the Electro-Magnetic Field
}

\author{
Sangwha-Yi* \\ Department of Math, Taejon University 300-716, South Korea
}

*Corresponding Author: Sangwha-Yi, Department of Math, Taejon University 300-716, South Korea

Abstract: In the special relativity theory, we study the gauge theory in the electro-magnetic field theory. Using that the Electro-magnetic potential is 4-vector, we treat the invariant potential. Electro-magnetic field theory's the gauge theory is expanded

Keywords: electro-magnetic potential, 4-vecter, the gauge theory

PACS Number: 003.30,41.20

1. INTRODUCTION

In the special relativity theory, electro-magnetic potential $(\phi, \vec{A})$ is 4-vector likely time-space

$\vec{E}=-\vec{\nabla} \phi-\frac{\partial \vec{A}}{c \partial t}, \vec{B}=\vec{\nabla} \times \vec{A}$

$\left(\frac{1}{c^{2}} \frac{\partial^{2}}{\partial t^{2}}-\nabla^{2}\right) A^{\alpha}=\frac{4 \pi}{c} j^{\alpha}=\frac{4 \pi}{c} \rho_{0} \frac{d x^{\alpha}}{d \tau}$

$\frac{1}{c} \frac{\partial}{\partial t}=\gamma\left(\frac{1}{c} \frac{\partial}{\partial t^{\prime}}-\frac{\vec{v}}{c} \cdot \vec{\nabla}^{\prime}\right), \vec{\nabla}=\vec{\nabla}^{\prime}-\gamma \frac{\vec{v}}{c} \frac{1}{c} \frac{\partial}{\partial t^{\prime}}-(1-\gamma) \frac{\vec{v}}{v^{2}} \cdot \vec{\nabla}^{\prime} \vec{v}$

$c t=\gamma\left(c t^{\prime}+\frac{\vec{v}}{c} \cdot \vec{x}^{\prime}\right), \quad \vec{x}=\vec{x}^{\prime}+\gamma \frac{\vec{v}}{c} c t^{\prime}-(1-\gamma) \frac{\vec{v} \cdot \vec{x}^{\prime}}{v^{2}} \vec{v}$

$\phi=\gamma\left(\phi^{\prime}+\frac{\vec{v}}{c} \cdot \vec{A}^{\prime}\right)$

$\vec{A}=\vec{A}^{\prime}+\gamma \frac{\vec{v}}{c} \phi^{\prime}-(1-\gamma) \frac{\vec{v} \cdot \vec{A}^{\prime}}{v^{2}} \vec{v}, \gamma=1 / \sqrt{1-\frac{v^{2}}{c^{2}}}$

\section{GaUge Transformation}

Gauge transformation of electro-magnetic potential $(\phi, \vec{A})$ of an inertial coordinate system $\mathrm{S}$ and electro-magnetic potential $\left(\phi^{\prime}, \vec{A}^{\prime}\right)$ of the other inertial coordinate system $S$ " is

$\phi \rightarrow \phi-\frac{1}{c} \frac{\partial \Lambda}{\partial t}, \vec{A} \rightarrow \vec{A}+\vec{\nabla} \Lambda$

$\Lambda$ is a function of $\mathrm{S}$

$\phi^{\prime} \rightarrow \phi^{\prime}-\frac{1}{c} \frac{\partial \Lambda^{\prime}}{\partial t^{\prime}}, \quad \vec{A}^{\prime} \rightarrow \vec{A}^{\prime}+\vec{\nabla}^{\prime} \Lambda^{\prime}$

$\Lambda^{\prime}$ is a function of $S^{\prime}$

Therefore, Eq (5), Eq (6) are used by Eq (7) and Eq (8)

$\phi-\frac{1}{c} \frac{\partial \Lambda}{\partial t}=\gamma\left\{\phi^{\prime}-\frac{1}{c} \frac{\partial \Lambda^{\prime}}{\partial t^{\prime}}+\frac{\vec{v}}{c} \cdot\left(\vec{A}^{\prime}+\vec{\nabla}^{\prime} \Lambda^{\prime}\right)\right\}$ 
$=\gamma\left\{\phi^{\prime}+\frac{\vec{v}}{c} \cdot \vec{A}^{\prime}\right\}+\gamma\left\{-\frac{1}{c} \frac{\partial \Lambda^{\prime}}{\partial t^{\prime}}+\frac{\vec{v}}{c} \cdot \vec{\nabla}^{\prime} \Lambda^{\prime}\right\}$

$\vec{A}+\vec{\nabla} \Lambda=\vec{A}^{\prime}+\vec{\nabla}^{\prime} \Lambda^{\prime}+\gamma \frac{\vec{v}}{c}\left(\phi^{\prime}-\frac{1}{c} \frac{\partial \Lambda^{\prime}}{\partial t^{\prime}}\right)-(1-\gamma) \frac{\vec{v}}{v^{2}} \cdot\left(\vec{A}^{\prime}+\vec{\nabla}^{\prime} \Lambda^{\prime}\right) \vec{v}$

$=\left\{\vec{A}^{\prime}+\gamma \frac{\vec{v}}{c} \phi^{\prime}-(1-\gamma) \frac{\vec{v}}{v^{2}} \cdot \vec{A}^{\prime} \vec{v}\right\}+\left\{\vec{\nabla}^{\prime} \Lambda^{\prime}+\gamma \frac{\vec{v}}{c}\left(-\frac{1}{c} \frac{\partial \Lambda^{\prime}}{\partial t^{\prime}}\right)-(1-\gamma) \frac{\vec{v}}{v^{2}} \cdot\left(\vec{\nabla}^{\prime} \Lambda^{\prime}\right) \vec{v}\right.$

In this time, if $\mathrm{Eq}(9), \mathrm{Eq}(10)$ are used by $\mathrm{Eq}(5), \mathrm{Eq}(6)$, the gauge function $\Lambda$ and $\Lambda^{\prime}$ 's relation is

$-\frac{1}{c} \frac{\partial \Lambda}{\partial t}=\gamma\left(-\frac{1}{c} \frac{\partial \Lambda^{\prime}}{\partial t^{\prime}}+\frac{\vec{v}}{c} \cdot \vec{\nabla}^{\prime} \Lambda^{\prime}\right)=\gamma\left(-\frac{1}{c} \frac{\partial}{\partial t^{\prime}}+\frac{\vec{v}}{c} \cdot \vec{\nabla}^{\prime}\right) \Lambda^{\prime}$

$\vec{\nabla} \Lambda=\vec{\nabla}^{\prime} \Lambda^{\prime}+\gamma \frac{\vec{v}}{c}\left(-\frac{1}{c} \frac{\partial \Lambda^{\prime}}{\partial t^{\prime}}\right)-(1-\gamma) \frac{\vec{v}}{v^{2}} \cdot\left(\vec{\nabla}^{\prime} \Lambda^{\prime}\right) \vec{v}$

$=\left[\vec{\nabla}^{\prime}+\gamma \frac{\vec{v}}{c}\left(-\frac{1}{c} \frac{\partial}{\partial t^{\prime}}\right)-(1-\gamma) \frac{\vec{v}}{v^{2}} \cdot\left(\vec{\nabla}^{\prime}\right) \vec{v}\right] \Lambda^{\prime}$

In this time, Eq (3) coincides Eq (11), Eq (12). Therefore a function $\Lambda$ of $S$ coincides $\Lambda^{\prime}$ of $S^{\prime}$.

Hence $\Lambda=\Lambda^{\prime}$.

Likely this upper case

$\phi^{2}-\vec{A} \cdot \vec{A}=\phi^{\prime 2}-\vec{A}^{\prime} \cdot \vec{A}^{\prime}$

$\mathrm{Eq}(13)$ is used by $\mathrm{Eq}(7), \mathrm{Eq}(8)$ of the gauge transformation. If we use $\mathrm{Eq}(3), \mathrm{Eq}$ (5), $\mathrm{Eq}(6)$ and $\mathrm{Eq}(11), \mathrm{Eq}(12)$,

$$
\begin{aligned}
& \left(\phi-\frac{1}{c} \frac{\partial \Lambda}{\partial t}\right)^{2}-(\vec{A}+\vec{\nabla} \Lambda) \cdot(\vec{A}+\vec{\nabla} \Lambda) \\
& =\left(\phi^{2}-\vec{A} \cdot \vec{A}\right)-2\left(\frac{1}{c} \frac{\partial \Lambda}{\partial t} \phi+\vec{A} \cdot \vec{\nabla} \Lambda\right)+\left[\frac{1}{c^{2}}\left(\frac{\partial \Lambda}{\partial t}\right)^{2}-\vec{\nabla} \Lambda \cdot \vec{\nabla} \cdot \Lambda\right] \\
& =\left(\phi^{\prime 2}-\vec{A}^{\prime} \cdot \vec{A}^{\prime}\right)-2\left(\frac{1}{c} \frac{\partial \Lambda^{\prime}}{\partial t^{\prime}} \phi^{\prime}+\vec{A}^{\prime} \cdot \vec{\nabla}^{\prime} \Lambda^{\prime}\right)+\left[\frac{1}{c^{2}}\left(\frac{\partial \Lambda^{\prime}}{\partial t^{\prime}}\right)^{2}-\vec{\nabla}^{\prime} \Lambda^{\prime} \cdot \vec{\nabla}^{\prime} \cdot \Lambda^{\prime}\right] \\
& =\left(\phi^{\prime}-\frac{1}{c} \frac{\partial \Lambda^{\prime}}{\partial t^{\prime}}\right)^{2}-\left(\overrightarrow{A^{\prime}+\vec{\nabla}^{\prime}} \Lambda^{\prime}\right) \cdot\left(\vec{A}^{\prime}+\vec{\nabla}^{\prime} \Lambda^{\prime}\right) \\
& \left(\phi^{2}-\vec{A} \cdot \vec{A}\right)=\left(\phi^{\prime 2}-\vec{A}^{\prime} \cdot \vec{A}^{\prime}\right), \\
& \left(\frac{1}{c} \frac{\partial \Lambda}{\partial t} \phi+\vec{A} \cdot \vec{\nabla} \Lambda\right)=\left(\frac{1}{c} \frac{\partial \Lambda^{\prime}}{\partial t^{\prime}} \phi^{\prime}+\vec{A}^{\prime} \cdot \vec{\nabla}^{\prime} \Lambda^{\prime}\right) \\
& \left(\frac{1}{c} \frac{\partial \Lambda}{\partial t}\right)^{2}-\vec{\nabla} \Lambda \cdot \vec{\nabla} \Lambda=\left(\frac{1}{c} \frac{\partial \Lambda^{\prime}}{\partial t^{\prime}}\right)^{2}-\vec{\nabla}^{\prime} \Lambda^{\prime} \cdot \vec{\nabla}^{\prime} \Lambda^{\prime}, \Lambda=\Lambda^{\prime}
\end{aligned}
$$

$\mathrm{Eq}(13)$ is invariant by $\mathrm{Eq}(14)$ about the gauge transformation, $\mathrm{Eq}(7), \mathrm{Eq}(8)$. .Eq (13) is invariant about the special relativistic transformation. Therefore, we can think the invariant electro-magnetic potential $\bar{\phi}$ likely the coordinate system's the invariant time.

$\bar{\phi}^{2}=\phi^{2}-\vec{A} \cdot \vec{A}=\phi^{12}-\vec{A}^{\prime} \cdot \vec{A}^{\prime}$

In $\mathrm{Eq}(2)$, electro-magnetic potential $A^{\alpha}$ transform likely the differential coordinate $d x^{\alpha}$ 
$d \tau^{2}=d t^{2}-\frac{1}{c^{2}}(d \vec{x} \cdot d \vec{x})$

$d \tau^{2}=d t^{2}\left(1-\frac{u^{2}}{c^{2}}\right), \frac{d \vec{x}}{d t}=\vec{u}$

$\bar{\phi}^{2}=\phi^{2}\left(1-\frac{u^{2}}{c^{2}}\right), \bar{\phi}=\phi \sqrt{1-\frac{u^{2}}{c^{2}}}$

$\frac{\vec{A}}{\phi}=\frac{\vec{u}}{c}, \vec{A}=\frac{\vec{u}}{c} \phi$

An example of Eq (22)'s potential is Lienard-Wiechert potential that made by moving point charge. $[3,4]$

In the inertial coordinate system and the other inertial coordinate system,

$\bar{\phi}^{2}=\phi^{2}\left(1-\frac{u^{2}}{c^{2}}\right)=\phi^{\prime 2}\left(1-\frac{u^{\prime 2}}{c^{2}}\right), \frac{d \vec{x}}{d t}=\vec{u}, \frac{d \vec{x}^{\prime}}{d t^{\prime}}=\vec{u}^{\prime}$

$\frac{\vec{A}}{\phi}=\frac{\vec{u}}{c}, \vec{A}=\frac{\vec{u}}{c} \phi, \quad \frac{\vec{A}^{\prime}}{\phi^{\prime}}=\frac{\vec{u}^{\prime}}{c}, \vec{A}^{\prime}=\frac{\vec{u}^{\prime}}{c} \phi^{\prime}$

$d \vec{x}=d \vec{x}^{\prime}+\gamma \frac{\vec{v}}{c} c d t^{\prime}-(1-\gamma) \frac{\vec{v} \cdot d \vec{x}^{\prime}}{v^{2}} \vec{v}, d t=\gamma\left(d t^{\prime}+\frac{\vec{v}}{c^{2}} \cdot d \vec{x}^{\prime}\right)$

$\vec{u}=\frac{d \vec{x}}{d t}=\frac{1}{\gamma} \frac{\vec{u}^{\prime}+\gamma \vec{v}-(1-\gamma) \frac{\vec{v} \cdot \vec{u}^{\prime}}{v^{2}} \vec{v}}{\left(1+\frac{\vec{v}}{c^{2}} \cdot \vec{u}^{\prime}\right)}, \vec{u}^{\prime}=\frac{d \vec{x}^{\prime}}{d t^{\prime}}, \phi=\gamma\left(\phi^{\prime}+\frac{\vec{v}}{c} \cdot \vec{A}^{\prime}\right)$

$\vec{A}=\frac{\vec{u}}{c} \phi=\frac{1}{\gamma}\left[\frac{\vec{u}^{\prime}+\vec{v}-(1-\gamma) \frac{\vec{v} \cdot \vec{u}^{\prime}}{v^{2}} \vec{v}}{\left(1+\frac{\vec{v}}{c^{2}} \cdot \vec{u}^{\prime}\right)}\right] \cdot \gamma \frac{1}{c}\left[\phi^{\prime}+\frac{\vec{v}}{c} \cdot \vec{A}^{\prime}\right), \quad \vec{A}^{\prime}=\frac{\vec{u}^{\prime}}{c} \phi^{\prime}$

$=\frac{1}{\gamma}\left[\frac{\vec{u}^{\prime}+\gamma \vec{v}-(1-\gamma) \frac{\vec{v} \cdot \vec{u}^{\prime}}{v^{2}} \vec{v}}{\left(1+\frac{\vec{v}}{c^{2}} \cdot \vec{u}^{\prime}\right)}\right] \cdot \gamma \frac{1}{c} \phi^{\prime}\left[1+\frac{\vec{v}}{c} \cdot \frac{\vec{u}^{\prime}}{c}\right]$

$=\frac{\vec{u}^{\prime}}{c} \phi^{\prime}+\gamma \vec{v} \frac{\phi^{\prime}}{c}-(1-\gamma) \frac{\vec{v}}{v^{2}} \cdot\left(\vec{u}^{\prime} \frac{\phi^{\prime}}{c}\right) \vec{v}, \vec{A}^{\prime}=\frac{\vec{u}^{\prime}}{c} \phi^{\prime}$

$=\vec{A}^{\prime}+\gamma \frac{\vec{v}}{c} \phi^{\prime}-(1-\gamma) \frac{\vec{v} \cdot \vec{A}^{\prime}}{v^{2}} \vec{v}, \gamma=1 / \sqrt{1-\frac{v^{2}}{c^{2}}}$

According to Eq (22), electro-magnetic field is

$$
\begin{aligned}
& \vec{E}=-\vec{\nabla} \phi-\frac{\partial \vec{A}}{c \partial t}=-\vec{\nabla} \phi-\frac{\partial}{c \partial t}\left(\frac{\vec{u}}{c} \phi\right)=-\vec{\nabla} \phi-\frac{\phi}{c} \frac{\partial \vec{u}}{c \partial t}-\frac{\vec{u}}{c} \frac{\partial \phi}{c \partial t} \\
& \vec{B}=\vec{\nabla} \times \vec{A}=\vec{\nabla} \times\left(\frac{\vec{u}}{c} \phi\right)=\phi\left(\vec{\nabla} \times \frac{\vec{u}}{c}\right)-\frac{\vec{u}}{c} \times \vec{\nabla} \phi
\end{aligned}
$$

Electro-magnetic potential equation is in $\mathrm{Eq}(2)$,

$\left(\frac{1}{c^{2}} \frac{\partial^{2}}{\partial t^{2}}-\nabla^{2}\right) \phi=\frac{4 \pi}{c} j^{0}=4 \pi \rho=\frac{4 \pi}{c} \rho_{0} \frac{d x^{0}}{d \tau}=\frac{4 \pi}{c} \rho_{0} \frac{c d t}{d \tau}=4 \pi \bar{\gamma} \rho_{0}$ 


$$
\begin{aligned}
& j^{0}=c \rho, \rho=\bar{\gamma} \rho_{0}, \quad \bar{\gamma}=\frac{1}{\sqrt{1-\frac{u^{2}}{c^{2}}}} \\
& \left(\frac{1}{c^{2}} \frac{\partial^{2}}{\partial t^{2}}-\nabla^{2}\right) \vec{A}=\left(\frac{1}{c^{2}} \frac{\partial^{2}}{\partial t^{2}}-\nabla^{2}\right) \phi \frac{\vec{u}}{c} \\
& =\frac{\vec{u}}{c}\left(\frac{1}{c^{2}} \frac{\partial^{2}}{\partial t^{2}}-\nabla^{2}\right) \phi+\phi\left(\frac{1}{c^{2}} \frac{\partial^{2}}{\partial t^{2}}-\nabla^{2}\right) \frac{\vec{u}}{c},\left(\frac{1}{c^{2}} \frac{\partial^{2}}{\partial t^{2}}-\nabla^{2}\right) \frac{\vec{u}}{c}=\overrightarrow{0} \\
& =\frac{\vec{u}}{c}\left(\frac{1}{c^{2}} \frac{\partial^{2}}{\partial t^{2}}-\nabla^{2}\right) \phi,\left(\frac{1}{c^{2}} \frac{\partial^{2}}{\partial t^{2}}-\nabla^{2}\right) \phi=4 \pi \rho \\
& =\frac{4 \pi}{c} \rho \vec{u}=\frac{4 \pi}{c} \vec{j} \quad \vec{j} \quad, \quad \vec{j}=\rho \vec{u}, \rho=\bar{\gamma} \rho_{0}, \quad \overline{1} \\
& \vec{A}=\frac{\vec{u}}{c} \phi \quad, \sqrt{1-\frac{u^{2}}{c^{2}}}
\end{aligned}
$$

$\operatorname{By} \operatorname{Eq}(21)$, electro-magnetic invariant potential $\bar{\phi}$ is

$$
\bar{\gamma} \bar{\phi}=\phi, \bar{\gamma}=\frac{1}{\sqrt{1-\frac{u^{2}}{c^{2}}}}
$$

Therefore by Eq (25) and Eq (27), electro-magnetic invariant potential $\bar{\phi}$ 's equation is

$$
\begin{aligned}
& \left(\frac{1}{c^{2}} \frac{\partial^{2}}{\partial t^{2}}-\nabla^{2}\right) \bar{\gamma} \bar{\phi}=4 \pi \bar{\gamma} \rho_{0} \\
& \left(\frac{1}{c^{2}} \frac{\partial^{2}}{\partial t^{2}}-\nabla^{2}\right) \bar{\phi}=4 \pi \rho_{0}
\end{aligned}
$$

\section{CONCLUSION}

About the gauge functions $\Lambda$ and $\Lambda^{\prime}$, if we use Eq (17),

$$
\left(\frac{1}{c} \frac{\partial \Lambda}{\partial t}\right)^{2}-\vec{\nabla} \Lambda \cdot \vec{\nabla} \Lambda=\left(\frac{1}{c} \frac{\partial \Lambda^{\prime}}{\partial t^{\prime}}\right)^{2}-\vec{\nabla}^{\prime} \Lambda^{\prime} \cdot \vec{\nabla}^{\prime} \Lambda^{\prime}, \quad \Lambda=\Lambda^{\prime}
$$

Lorentz gauge is

$$
\frac{1}{c} \frac{\partial \phi}{\partial t}+\vec{\nabla} \cdot \vec{A}=0, \frac{1}{c} \frac{\partial \phi^{\prime}}{\partial t^{\prime}}+\vec{\nabla}^{\prime} \cdot \vec{A}^{\prime}=0
$$

Therefore the gauge functions $\Lambda$ and $\Lambda^{\prime}$ satisfy $\mathrm{Eq}(31)$

$$
\left(\frac{1}{c^{2}} \frac{\partial^{2}}{\partial t^{2}}-\nabla^{2}\right) \Lambda=\left(\frac{1}{c^{2}} \frac{\partial^{2}}{\partial t^{\prime 2}}-\nabla^{\prime 2}\right) \Lambda^{\prime}=0(31), \Lambda=\Lambda^{\prime}
$$




\section{REFERENCES}

[1] A.Miller,Albert Einstein's Special Theory of Relativity(Addison-Wesley Publishing Co., Inc., 1981)

[2] W.Rindler,Special Relativity(2nd ed., Oliver and Boyd,Edinburg, 1966)

[3] David J.Griffiths,Introduction to Electrodynamics(2nd ed.,Prentice Hall)

[4] John R.Reitz,Frederick J.Milford,Robert W.Christy,Foundations of ElectroMagnetic theory(3th ed,.)

[5] William H.Hayt,Jr,Engineering electromagnetics(5th ed.,Mcgraw-hill,1990)

Citation: Sangwha-Yi, (2019). Gauge Theory's Expansion in the Electro-Magnetic Field. International Journal of Advanced Research in Physical Science (IJARPS) 6(12), pp.1-5, 2019.

Copyright: (C) 2019 Authors, This is an open-access article distributed under the terms of the Creative Commons Attribution License, which permits unrestricted use, distribution, and reproduction in any medium, provided the original author and source are credited. 